See discussions, stats, and author profiles for this publication at: https://www.researchgate.net/publication/307920422

\title{
Mitigation of Erroneous Oscillations in Electromagnetic Transient Simulations using Analogue Filter Theory
}

Article in IET Science Measurement ? Technology · September 2016

DOI: 10.1049/iet-smt.2016.0166

CITATION

4 authors:

Sergio Kurokawa

São Paulo State University

170 PUBLICATIONS 440 CITATIONS

SEE PROFILE

A. Anderson Ricardo Justo de Araujo

São Paulo State University

22 PUBLICATIONS 11 CITATIONS

SEE PROFILE
Ailton Akira Shinoda

São Paulo State University

61 PUBLICATIONS 223 CITATIONS

SEE PROFILE

Eduardo C. M. Costa

University of São Paulo

71 PUBLICATIONS 209 CITATIONS

SEE PROFILE

Some of the authors of this publication are also working on these related projects:

Desenvolvimento de estudos em compostos de borracha de silicone com enfase em isolamento elétrico View project

Security on local area networks using Openflow Protocol. View project 


\title{
Mitigation of erroneous oscillations in electromagnetic transient simulations using analogue filter theory
}

ISSN 1751-8822

Received on 18th April 2016 Revised on 22nd August 2016 Accepted on 31st August 2016 doi: 10.1049/iet-smt.2016.0166 www.ietdl.org

\author{
Anderson R. J. Araújo ${ }^{1}$, Sérgio Kurokawa ${ }^{1}$, Ailton A. Shinoda ${ }^{1}$, Eduardo C. M. Costa ${ }^{\circledR}$ \\ ${ }^{1}$ Department of Electrical Engineering, Faculty of Engineering of Ilha Solteira, State University of São Paulo - FEIS/UNESP, \\ Ilha Solteira, Brazil \\ ${ }^{2}$ Department of Energy and Automation Engineering, Polytechnic School of the University of São Paulo - PEA/EPUSP, São Paulo, Brazil \\ $凶$ E-mail: educosta@pea.usp.br
}

\begin{abstract}
An efficient and simplified procedure is proposed for the reduction of high-frequency oscillations and erroneous magnitude peaks in electromagnetic transient simulations in power transmission systems modelled by the lumped electric parameters approach. This procedure consists of the inclusion of analogue filters in the equivalent representation of multiconductor transmission lines without modifying its electromagnetic propagation characteristics. The analogue filter modelling is conducted as a function of the line length and line parameters. The proposed simulation methodology is applied directly in the line modelling, which means that the filtering/correction process represents a real-time process during simulations, without post-processing filtering techniques using digital filters or variations in the solution methods. The results obtained directly in the time domain by the proposed modelling/simulation methodology are compared with simulations obtained from well-established line models using the numerical Laplace transform and the Bergeron method.
\end{abstract}

\section{Introduction}

The electromagnetic performance of power systems can be basically predicted from two methods: experimentation or simulations. The first method relies on by experimental tests, usually based on international standards, or empirical data measured in real power systems, which are restricted to specific operational and environmental conditions (e.g. the analysis of transmission lines during electromagnetic transients occurring due to atmospheric discharge or switching operations). The second analysis method is based on simulations using analogue or computational tools. Until the 1960s, power system simulations were performed using analogue simulators, which basically perform power system modelling by equivalent electric circuits in reduced scale. These simulators are well known in the technical literature as transient network analysers [1,2]. After 1970, based on the continuous advances in the processing resources of digital computers, several versions of the well-known electromagnetic transient program (EMTP) were developed for commercial and academic applications [3]. Currently, there are computers capable of performing simulations in real operation time, even considering fast electromagnetic transients. These dedicated computers are commercially known as real-time digital simulators [4].

The revolution in power system simulation is mainly attributed to the on-going progress in the improvements in computational processing due to hardware; however, this revolution was also possible because of many contributions from new simulation techniques and power system modelling during the recent decades. Simulation techniques using different integration methods were proposed to solve transmission line models in the time domain, improving the accuracy of the results and computational performance $[5,6]$. Modelling techniques were also proposed to improve the multiconductor line representation using modal transformation matrices and alternative methods to represent the mutual parameters between phases by using lumped parameters, without modal decoupling [7]. Multiconductor line modelling by lumped parameters and modal decoupling is a well-established approach in the technical literature on transmission line modelling (TLM). This approach consists basically in decoupling an $m$-phase line into $m$ independent propagation modes, which can be modelled as $m$ single-phase lines based on the lumped-parameters representation [7].

The representation of the line as an equivalent $\pi$ circuits produces a system of differential equations, which can be solved using numeric integration methods $[8,9]$. Although line representation by equivalent electric circuits has advantages compared with other models in the frequency domain (e.g. direct development in the time domain and the inclusion of time-variable elements in transient simulations), some inaccuracies are observed during simulations because of high-frequency oscillations that result from the line modelling and numerical integration methods. Some inaccuracies depend on the number of $\pi$ circuits per unit of length and have been previously discussed in the technical literature, along with methods to suppress these spurious oscillations [9].

A first solution for the numerical oscillations in TLM was proposed based on the use of two integration methods during simulations in the EMTP, alternating the trapezoidal and Euler rules through the numerical integration process. This technique suppresses the numerical oscillations by providing critical damping of the discontinuity within one integration step, $\Delta t$, of the simulation. The critical damping adjustment is achieved through two $\Delta t / 2$ using the backward Euler rule [10]. A second procedure for suppressing the numerical oscillations is applied directly in the line modelling using digital filtering techniques [11, 12]. This second solution can be considered as a post-processing method, as it is applied after the simulation and numerical integration processes. The method using two integration rules shows a better computational performance, whereas the method based on digital filtering simplifies the computational algorithm. However, the principal difference between the two methods for mitigating numerical oscillations is that the first method is applied to the numeric solution, while the second is applied directly in the line modelling. In this context, a multiconductor line model is proposed based on the lumped-parameters approach, where the numerical oscillations are suppressed by the inclusion of analogue filters directly in the line model. The modelling of the proposed analogue filter is given as a function of the line length and number of equivalent $\pi$ circuits in the cascade. A significant mitigation of 
the erroneous oscillations is obtained using only a single integration method without post-processing techniques (digital filtering), which usually result in shifts in successive wave reflections during the transient state $[11,12]$. Numerical methods have been widely used to simulate electromagnetic transients in transmission lines in the time as well as in the frequency domain. The principal methods in the technical literature are as follows: the finite-difference time-domain method [13], the method of moments [14], the method of characteristics [15] and the time-domain finite-element method for non-uniform [16], or multiconductor lines [17]. Based on the state of the art, this research proposes the inclusion of analogue low-pass filters in lumped-parameter line model to mitigate spurious oscillations in electromagnetic transient simulations in the time domain. The proposed line model is validated from simulations using the numerical Laplace transform (NLT) and other well-established line models available in the EMTP [18, 19]. In addition, the frequency response of the proposed line model, with and without the low-pass filtering technique, is evaluated to provide complementary information on the numerical oscillation suppression and eventual variations in the frequency-domain behaviour of the transmission line during electromagnetic transient simulations.

\section{Conventional line representation by lumped parameters}

The representation of a line by a cascade of $\pi$ circuits is a well-established technique in TLM for electromagnetic transient analyses. This line model is basically described in Fig. 1 [5] where a line segment is represented by a $\pi$ circuits cascade.

Each line section is modelled using the lumped resistance $R$ and inductance $L$ to represent the longitudinal impedance, whereas the transversal parameters are represented by lumped capacitances and conductances, $C$ and $G$, respectively. The terms $R, L, C$ and $G$ are calculated from the line parameters per unit of length $\ell$ and number of $\pi$ sections $n$, as described in the following equation

$$
R=R^{\prime} \frac{\ell}{n} ; \quad L=L^{\prime} \frac{\ell}{n} ; \quad C=C^{\prime} \frac{\ell}{n} ; \quad G=G^{\prime} \frac{\ell}{n}
$$

The terms $R^{\prime}$ and $L^{\prime}$ are the resistance and inductance per unit of length, respectively, and $C^{\prime}$ and $G^{\prime}$ are the transversal capacitance and conductance per unit of length, respectively. Considering a transmission line segmented into $m \pi$ sections (Fig. 1), the differential equations for the currents through the impedances and the voltages on the transversal admittances can be easily represented by the state matrices, as the transmission line is represented by a system of first-order differential equations and solved using numerical integration methods [5-7]. In this context, the lumped-parameters model presents several advantages, such as the modelling of time-variable and non-linear systems [8]. However, the solution of the state equations commonly presents numerical oscillations that affect the exact prediction of the voltage and current peaks in electromagnetic transient simulations. The numerical oscillations, which result from the numerical integration methods, can be observed in the transient simulations shown in Fig. $2 b$.

The voltage transients in Fig. $2 b$ are simulated from a single-phase transmission line connected at the sending end to a DC source and at the receiving end to a generic load represented by a resistance of $2000 \Omega$, as described in Fig. $2 a$. A switching impulse is simulated

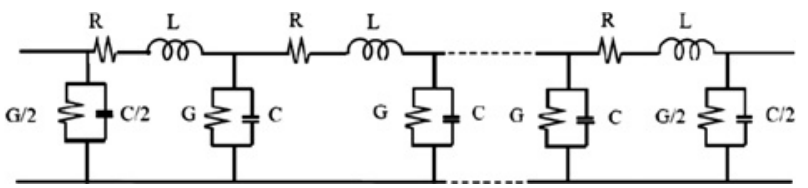

Fig. 1 Line represented by lumped parameters when the switch at the sending end is closed at $t=0$. The line parameters per unit length are as follows: $R^{\prime}=0.05 \Omega / \mathrm{km}, L^{\prime}=1.7$ $\mathrm{mH} / \mathrm{km}, G^{\prime}=0.556 \mu \mathrm{S} / \mathrm{km}$ and $C^{\prime}=6.58 \eta \mathrm{F} / \mathrm{km}$. Curves 1 and 2 are the voltage transients at the receiving end of the line as represented by 20 and 100 equivalent $\pi$ sections, respectively. The same transient condition is simulated by the line representation in the frequency domain using NLT, as described by curve 3 . The erroneous oscillations result in transient voltage peaks $\sim 20 \%$ greater than the simulations using NLT. When the number of $\pi$ circuits is increased, the distributed parameters of the line are better represented, and therefore the results obtained from the lumped-parameters model and using NLT (distributed parameters) are closer, as shown in Fig. 2. Another observation regarding the lumped-parameters modelling of the line is the relationship of the propagation time $2 T$, the number of $\pi$ circuits in the line representation and the frequency of the numerical oscillations. The constant $T$ is the propagation time of the electromagnetic wave from the sending to the receiving end. The constant $2 T$ represents the time for the electromagnetic wave to propagate from the sending to the receiving end and reflect back to the sending end. Fig. 3 compares the maximum voltage peak of the numerical oscillations for a transmission line represented by lumped parameters, as a function of the line length, and the line model represented by distributed parameters using NLT (reference model).

The transient voltage peaks are simulated considering the two line models for a line length varying from 10 to $500 \mathrm{~km}$. The results obtained from the lumped-parameters line model show transient voltage peaks $25 \%$ greater than the corresponding values simulated using NLT. Fig. $2 b$ shows that the frequency of the numerical oscillations varies as a function of the number of $\pi$ circuits in the line representation. Another observation is that the frequency of oscillations at $0.4 \mathrm{~ms}$ is different from the frequency at $0.9 \mathrm{~ms}$. This information is necessary to define the correlation between the number of $\pi$ sections and the line length. In Fig. $2 b, \tau$ represents the period of the first oscillation, so the maximum frequency for the spurious oscillations will be defined as follows

$$
f_{\mathrm{S}}=\frac{1}{\tau}
$$

Thus, the frequency at $0.4 \mathrm{~ms}$ can be assumed as a reference for calculating the maximum frequency values of the erroneous oscillations and represents important information for calculation of the analogue filter parameters. Fig. 4 shows the frequency of the numerical oscillations $\left(f_{\mathrm{S}}\right)$ as a function of line length $(\ell)$ and the number of $\pi$ circuits $(m)$ in the cascade. Term $k$ is the relationship between the number of equivalent $\pi$ circuits and line length

$$
k=\frac{m}{\ell}
$$

The maximum frequency of the spurious oscillations $\left(f_{\mathrm{S}}\right)$, considering the line represented by lumped parameters, is analysed for different values of $k$ and line lengths from $100 \mathrm{up}$ to $500 \mathrm{~km}$.

The set of curves in Fig. 4 shows that the frequency of the numerical oscillations is practically constant with line length (continuous curves), varying only for different values of the relationship $k$. Thus, a maximum frequency $f_{\max }$ can be defined to filter all frequencies for $k>0.1$, for example. The calculation of the cut-off frequency will be approached step-by-step in the next sections.

\section{Frequency analysis of simulations using the NLT}

The same single-phase line in Fig. $2 a$ is connected at the sending end to a voltage source $(t=0)$ represented by a cosine function with a peak magnitude of 1 p.u., as described in Fig. $5 a$. The voltage transient at the receiving end of the line is shown in Fig. $5 b$. The 

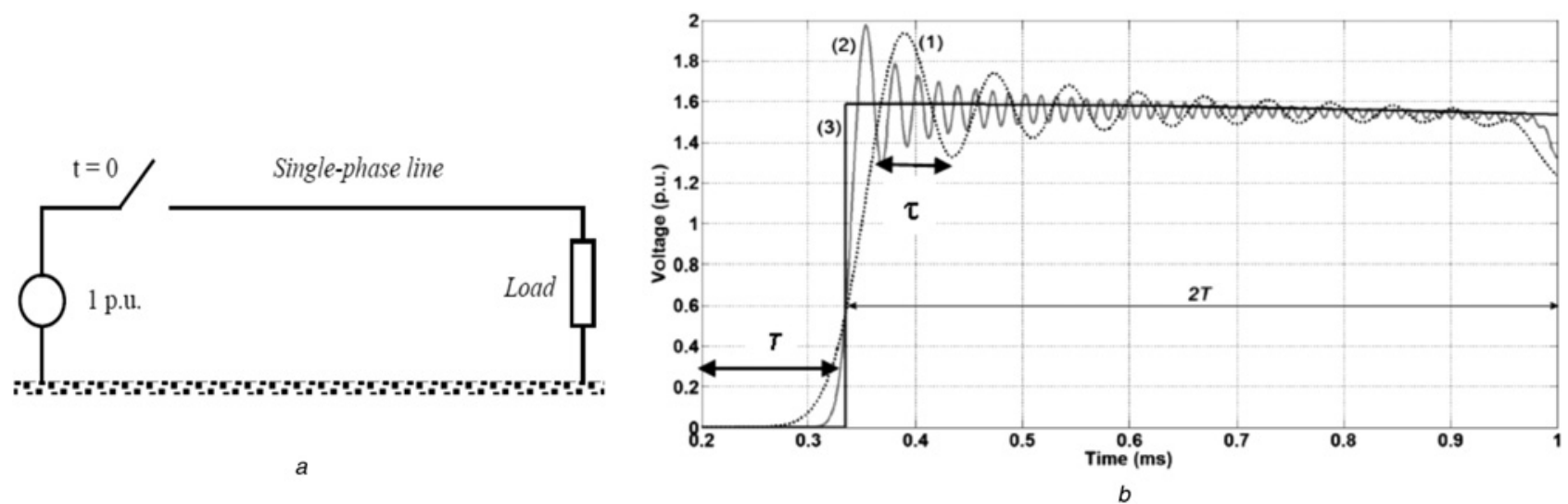

Fig. 2 Numerical oscillations resulting from the numerical integration methods

$a$ Single-phase line used for the transient analyses

$b$ Simulations using the lumped-parameters representation with 20 and 100 equivalent circuits (curves 1 and 2, respectively) and NLT (curve 3 )

transient simulation is conducted using the line model by distributed parameters and the line model by NLT.

The constant $T$ is the propagation time from the sending end to the receiving end, which can be calculated as a function of the geometrical and physical characteristics of the line. Considering that the wave speed in the real transmission line can be approximated by a lossless transmission line, it yields

$$
v=\frac{1}{\sqrt{L C}}
$$

In (4), $L$ and $C$ are the inductance and capacitance per unit length in the line represented by its distributed parameters. The propagation time $T$ can be calculated as follows

$$
T=\frac{\ell}{v}=\ell \sqrt{L C}
$$

In (5), the term $\ell$ is the line length. As the propagation time $T$ is calculated, the damped periodic function that represents the voltage $V_{\mathrm{R}}(t)$ at the receiving end (Fig. $\left.5 b\right)$ can be expressed as follows

$$
V_{\mathrm{R}}(t)=\left[\cos (2 \pi f(t-T))+x(t-T) \mathrm{e}^{-a(t-T)}\right] u(t-T)
$$

The signal at the receiving end can also be expressed by a cosine function with fundamental frequency $f=60 \mathrm{~Hz}$, where $u(t)$ is an unitary step function shifted by $T$. The function $x(t)$ represents $a \pm$ 0.5 p.u. symmetric rectangular wave with a period of $4 T$ and the term $a$ is a damping coefficient. The function $x(t)$ can be

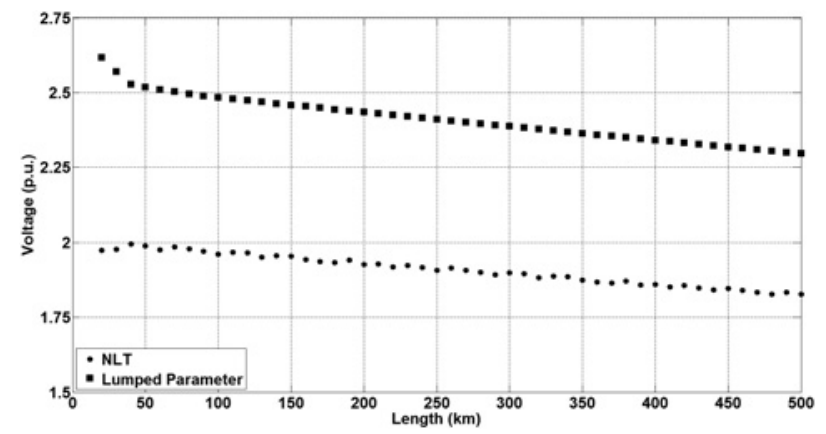

Fig. 3 First-peak magnitude at the receiving end of the line from models using NLT and using lumped parameters expressed as follows

$$
x(t)=\frac{2}{\pi} \sum_{n=0}^{\infty} \frac{(-1)^{n}}{2 n+1} \cos \left(2 \pi(2 n+1) f_{0} t\right)
$$

The fundamental frequency of $x(t)$ is denoted as $f_{0}$, which is also expressed as a function of the line parameters and from (4)

$$
f_{0}=\frac{1}{4 T}=\frac{1}{4 \ell \sqrt{L C}}
$$

The propagation speed through the conductor is expressed in the technical literature as a function of the line parameters and is conventionally, in most of cases, assumed as being the light speed. The symmetric rectangular wave has odd harmonic frequencies in its spectrum. Considering that low-pass filter will mitigate frequencies above of eighth harmonic $(n=8)$ from the rectangular wave $x(t)$, the maximum frequency, $f_{\max }$, for $x(t)$ is given by

$$
f_{\max }=17 f_{0}
$$

The filter should not cut off frequencies up to $f_{\max }$, as defined in (9). However, frequencies higher than the eighth harmonic component in $x(t)$ can be filtered during transient simulations. Harmonic components above the eighth harmonic have $<6 \%$ of the fundamental component magnitude and can be assumed to be the maximum frequency $f_{\max }$. In this context, the cut-off frequency in (9) can be restructured as a function of the line parameters and

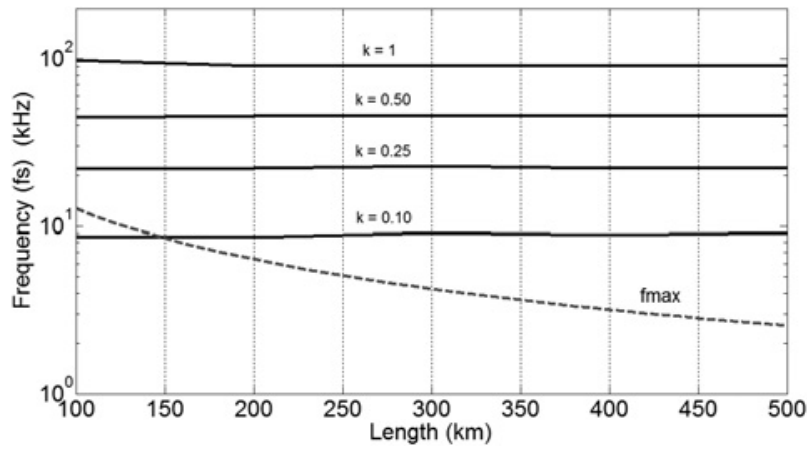

Fig. 4 Frequency of the numerical oscillations as a function of $k$ and line length 


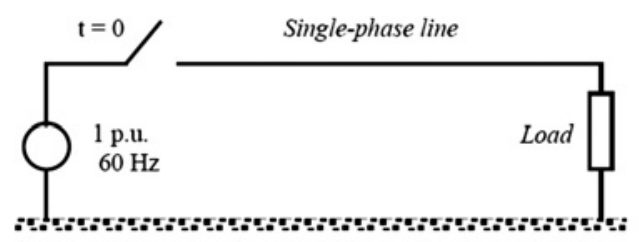

a

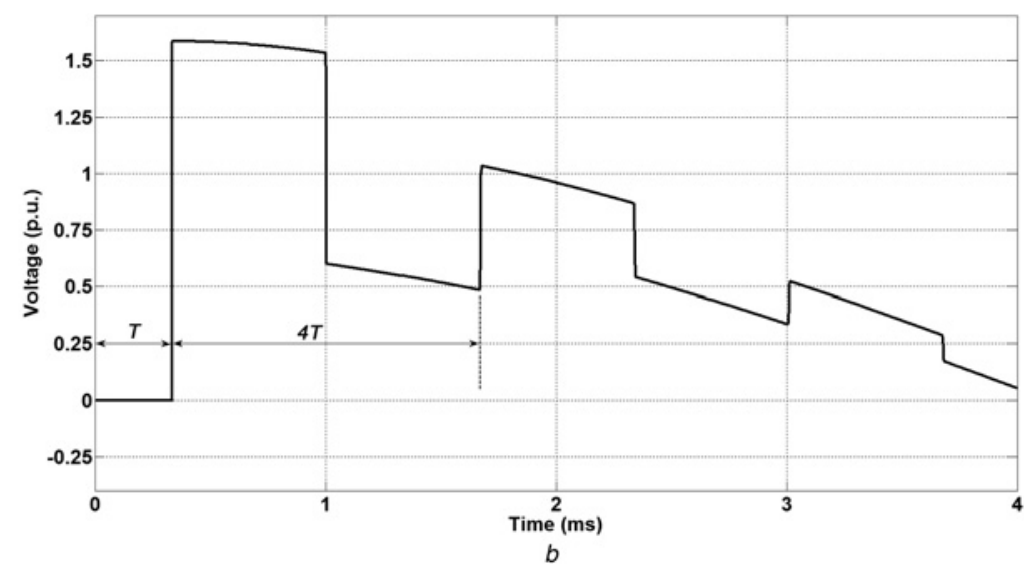

Fig. 5 Single-phase line and voltage transient at the receiving end of the line

$a$ Single-phase line used for the transient analyses

$b$ Voltage at the receiving end using the line model with NLT

length and given by

$$
f_{\max }=\frac{17}{4} \frac{1}{\ell \sqrt{L C}}
$$

As in (3), the maximum frequency in (10) is given as a function of the length $(\ell)$ and line parameters. Fig. 4 describes the numerical oscillation frequency $f_{\mathrm{S}}$ for different values of $k$ and maximum frequency $f_{\max }$. Fig. 4 shows that for $0.1 \leq k \leq 1$ and $150 \leq \ell \leq$ $500 \mathrm{~km}$, the maximum frequencies for the spurious oscillations are above the maximum frequency $f_{\max }$. If the frequency, as a function of $k$ and $\ell$, is constantly above the curve of $f_{\max }$ in Fig. 4, the same filtering procedure can be extended for transmission lines longer than $500 \mathrm{~km}$. As the line parameters and line length are known, the cut-off frequency $f_{\text {off }}$ can be determined, and the low-pass $\pi$ filter can be modelled and included in the line representation.

\section{Inclusion of analogue filters in the lumped parameters}

As shown in Fig. 2, line modelling by lumped parameters results in erroneous numeric oscillations during transient simulations. In this context, analogue filters can be integrated into the equivalent circuit by lumped parameters, directly in the time domain, to suppress the numerical oscillation without affecting the line response in the time and frequency domains. This paper proposes a systematic methodology for the calculation and inclusion of low-pass $\pi$ filters in multiconductor line models by lumped elements in the time domain. The equivalent electric circuits for the analogue filter and line representation are described in Fig. 6.
The cut-off frequency $f_{\text {off }}$ of the $\pi$ filter is defined when in frequency response (output signal) decays in $<3 \mathrm{~dB}$ [20]. Based on the concept of low-pass analogue filters, the cut-off frequency of the $\pi$ filter can be treated as a function of the resistance $R_{\mathrm{f}}$, inductance $L_{\mathrm{f}}$ and capacitance $C_{\mathrm{f}}$, as shown in Fig. 6a and expressed in the following equations

$$
\begin{gathered}
f_{\text {off }}=\frac{1}{2 \pi} \sqrt{\frac{\beta+\sqrt{\beta^{2}+4 L_{\mathrm{f}}^{2} C_{\mathrm{f}}^{2}}}{2 L_{\mathrm{f}}^{2} C_{\mathrm{f}}^{2}}} \\
\beta=C_{\mathrm{f}}^{2}\left(R_{\mathrm{f}}^{2}-2 L_{\mathrm{f}}^{2}\right)
\end{gathered}
$$

As the proposed filter must mitigate the spurious oscillations inherent of the lumped-parameters modelling, the cut-off frequency of the analogue low-pass filter $\left(f_{\text {off }}\right)$ must be less than the maximum frequency of the spurious oscillations $\left(f_{\mathrm{S}}\right)$, as expressed by

$$
f_{\text {off }}<f_{\mathrm{S}}
$$

In addition, the cut-off frequency for the low-pass filter must be greater than the maximum frequency $f_{\max }$, as the filtering should not affect frequencies up to $f_{\max }$

$$
f_{\max }<f_{\text {off }}<f_{\mathrm{S}}
$$

The frequency range in (14) depends on the line parameters, according to (4) and (10). The $\pi$ filters are located at the sending and receiving ends of each propagation model (Fig. 6b), as a multiconductor system can be easily modelled using modal decoupling techniques (modal transformation), as extensively

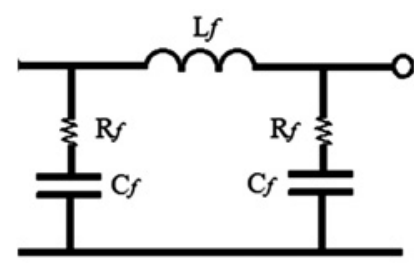

a

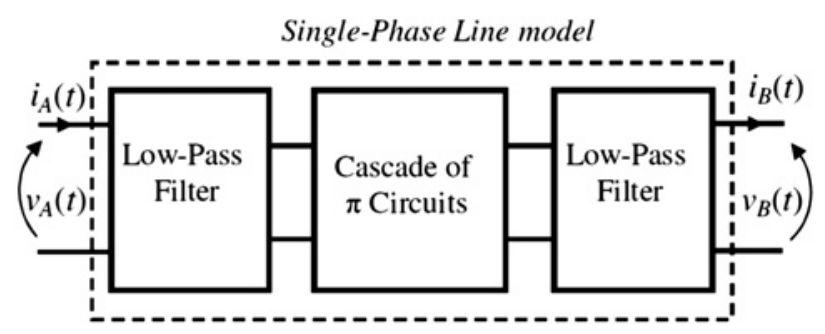

Fig. 6 Equivalent electric circuits for the analogue filter and line representation

$a$ Electric circuit of analogue low-pass $\pi$ filter

$b$ Transmission line model including low-pass filters for mitigation of the numerical oscillations 

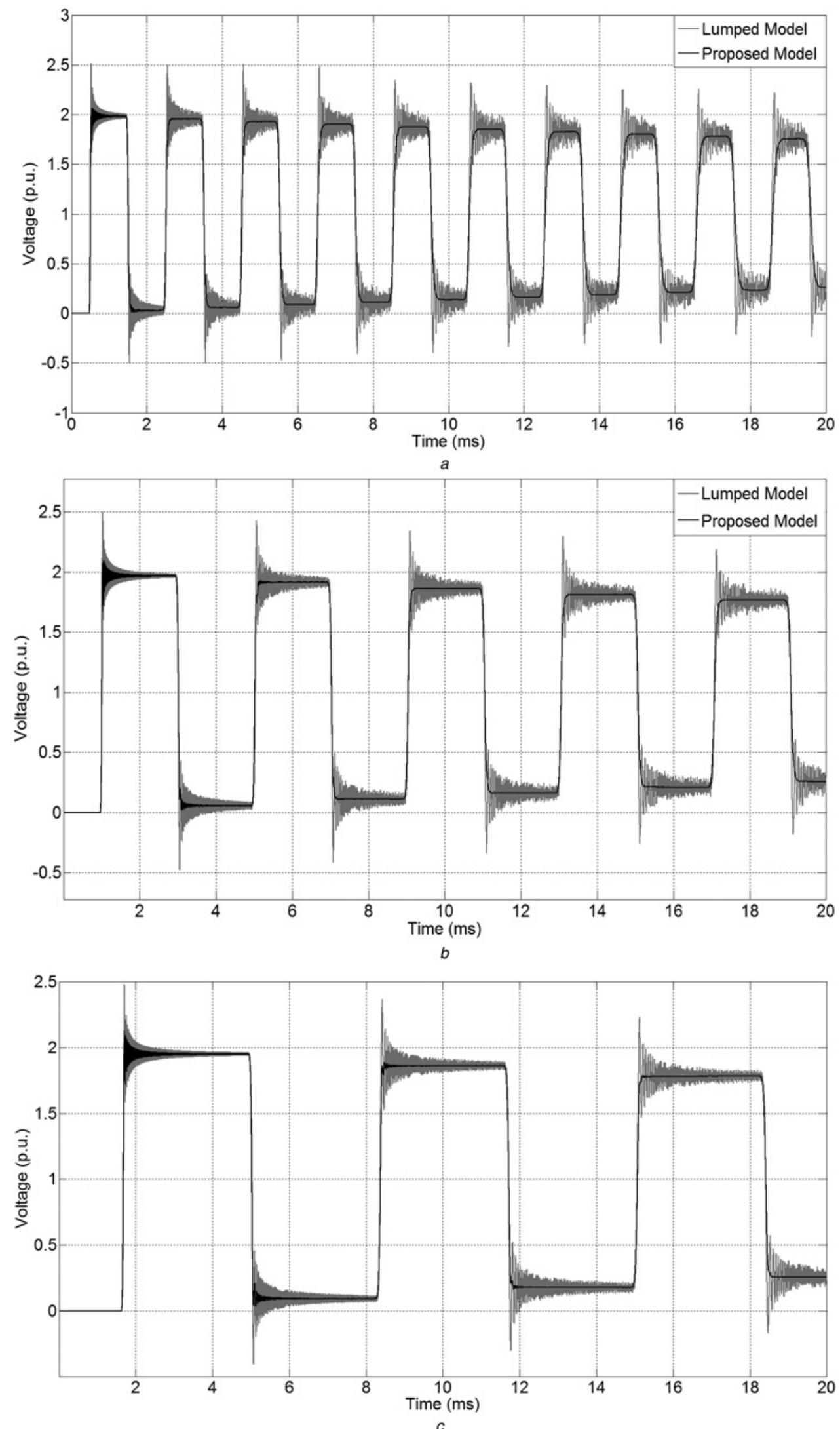

Fig. 7 Transient voltages at the receiving end of a single-phase line

$a$ Line with length of $150 \mathrm{~km}$

$b$ Line with length of $300 \mathrm{~km}$

$c$ Line with length of $500 \mathrm{~km}$

discussed in the technical literature on TLM $[7,8,11]$. The modal transformation decouples a three-phase transmission line into three independent propagation modes, i.e. each propagation mode can be represented as a single-phase line, where the low-pass $\pi$ filters are modelled as a function of the respective modal parameters and located at the two terminals, as described in Fig. $6 b$. 


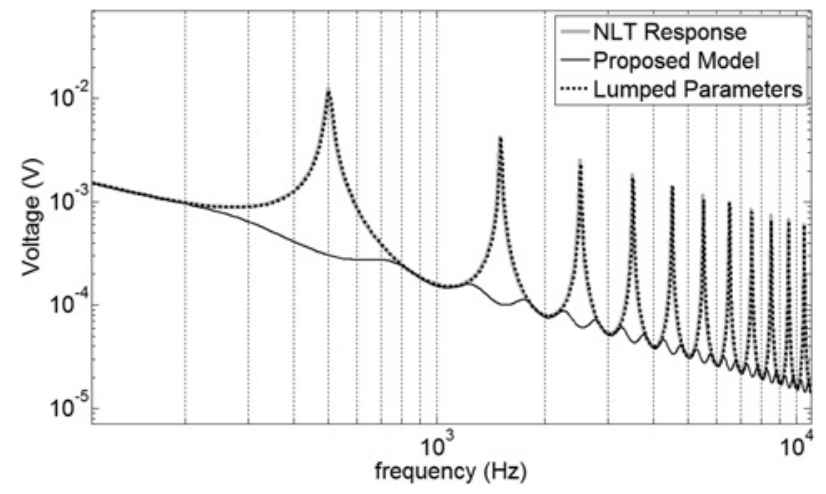

Fig. 8 Frequency responses for the $150 \mathrm{~km}$ transmission line represented by diverse models

Table 1 Harmonic frequencies and its peaks from the proposed model in frequency response

\begin{tabular}{lccc}
\hline Harmonic & Frequency, $\mathrm{Hz}$ & Peak $\left(\times 10^{-3}\right)$ & Ratio \\
\hline 1st & 500 & 0.306588 & 1.00000 \\
3rd & 1500 & 0.102308 & 0.33472 \\
5th & 2500 & 0.061516 & 0.20126 \\
7th & 3500 & 0.044072 & 0.14419 \\
9th & 4500 & 0.034405 & 0.11256 \\
11th & 5500 & 0.028265 & 0.09248 \\
13th & 6500 & 0.024018 & 0.07858 \\
15th & 7500 & 0.020900 & 0.06838 \\
17th & 8500 & 0.018506 & 0.06055 \\
\hline
\end{tabular}

\section{$5 \quad$ Electromagnetic transient simulations using the $\pi$ filter modelling}

As a first approach, transient simulations using the proposed technique are conducted for the same single-phase line in Fig. $2 a$, with length of 150,300 and $500 \mathrm{~km}$. The line parameters were previously described in Section 2 along with the voltage source and load at the line terminals. The voltage transients are simulated using the proposed line model with filtering and the conventional model by lumped parameters. The transient voltage profiles at the load terminal, simulated for single-phase lines 150, 300 and 500 $\mathrm{km}$ in length, are depicted in Fig. 7.

Simulations from both line models are conducted considering the relationship $k=1$ and $f_{\mathrm{S}}=100 \mathrm{kHz}$. The cut-off frequency for the low-pass filters is fixed at $f_{\text {off }}=60 \mathrm{kHz}$, and the low-pass $\pi$ filter parameters in Fig. $6 a$ are $L_{\mathrm{f}}=0.5 \mathrm{mH}, C_{\mathrm{f}}=10 \eta \mathrm{F}$ and $R_{\mathrm{f}}=800 \Omega$. Fig. 7 shows that the numerical oscillations are significantly reduced by the proposed filtering technique and line modelling.

The frequency response of a transmission line with a length of $150 \mathrm{~km}$, or a single propagation mode, is observed in Fig. 8. An unitary step ( 1 p.u.) is applied at the sending end of the line, and voltage transients at the receiving end are obtained from the line model using lumped parameters $(k=1)$, with and without the proposed filtering technique, and the line model using NLT.

Considering an electromagnetic propagation velocity of $\sim 300,000 \mathrm{~km} / \mathrm{s}$ and (5) and (8), the fundamental frequency is $f_{0}=$ $500 \mathrm{~Hz}$. The NLT response in Fig. 7 is a damped rectangular wave, as expressed in (7) by applying the Fourier series, where the amplitude of the components $1 /(2 n+1)$ decreases with the inverse of the harmonic frequency of $n$. Thus, the peaks in Fig. 8 occur at the odd harmonic frequencies (poles) in the NLT responses $\left(3 f_{0}=\right.$ $\left.1.500 \mathrm{~Hz}, 5 f_{0}=2.500 \mathrm{~Hz}, 7 f_{0}=3.500 \mathrm{~Hz}, \ldots\right)$. Analogously, even though the frequency responses for the NLT and proposed model (dotted curve) are not similar in Fig. 8, the proposed model maintains the same format as the NLT response in the time domain, as shown in Fig. 7. Table 1 shows some odd harmonic frequencies and the corresponding peaks from Fig. 8 .

The ratio of the $n$th harmonic component and the fundamental component follows approximately the coefficient relation $1 /(2 n+$ 1). For example, the ratio of the third and first harmonics is $0.33472 ; 1 / 3$, and the ratio of the fifth and first harmonics is $0.20126 ; 1 / 5$. Based on this observation, the filtering technique and line model suppress the spurious numerical oscillations while preserving the proportions between the harmonic components that compose the line response in the frequency domain.

\section{Three-phase line model using the proposed method}

The proposed modelling technique can be extended to represent three-phase lines by lumped parameters using modal decoupling, as is well established in the technical literature on TLM [7, 8, 12]. A three-phase line can be decoupled into three independent propagation modes, which can be modelled as three single-phase lines using the lumped-parameters approach and the proposed filtering technique, as shown in Fig. 6. Conventionally, the modal decoupling is achieved using a modal transformation matrix consisting of real and constant terms or complex and frequency-variable terms in the case of frequency-dependent line models for simulation of fast electromagnetic transients [7]. In the proposed line model, the transient simulations are conducted in the time domain for each propagation mode separately, i.e. the current
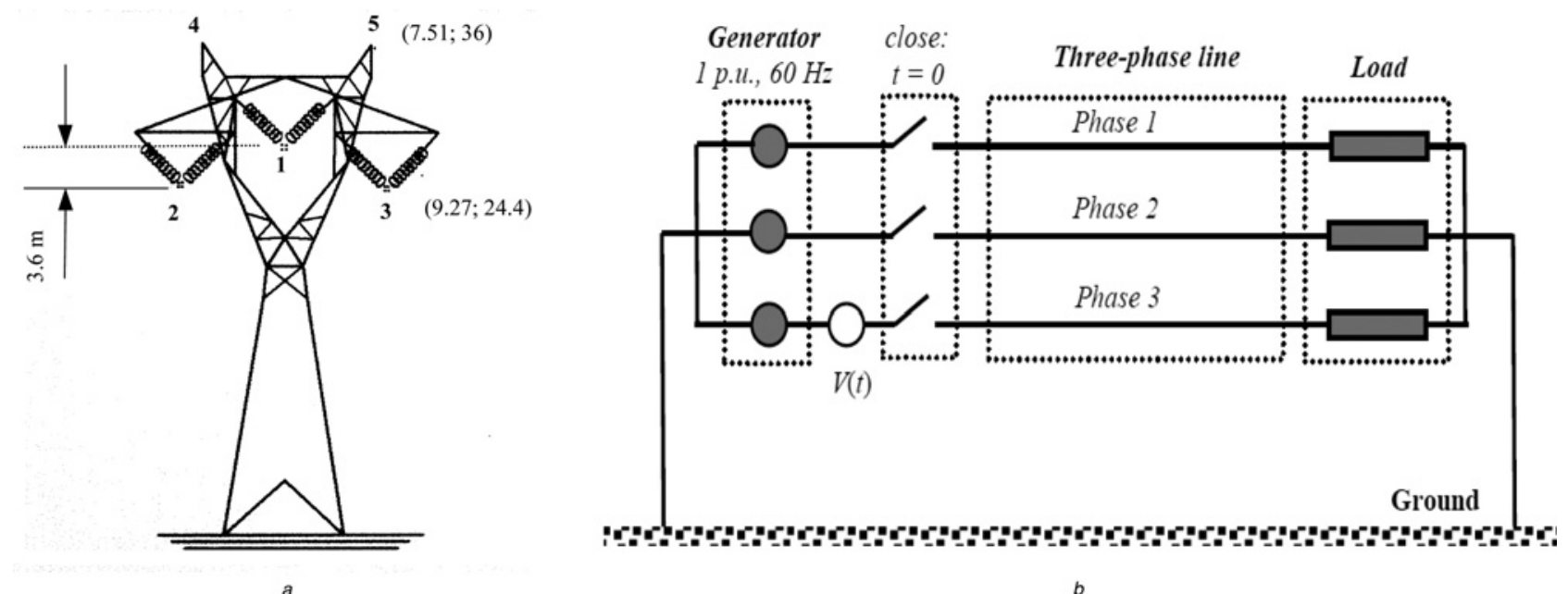

Fig. 9 Transmission tower profile and switching simulation of a $440 \mathrm{kV}$ transmission system

$a$ Transmission tower profile and its dimensions

$b$ Switching simulation of a $440 \mathrm{kV}$ transmission system 


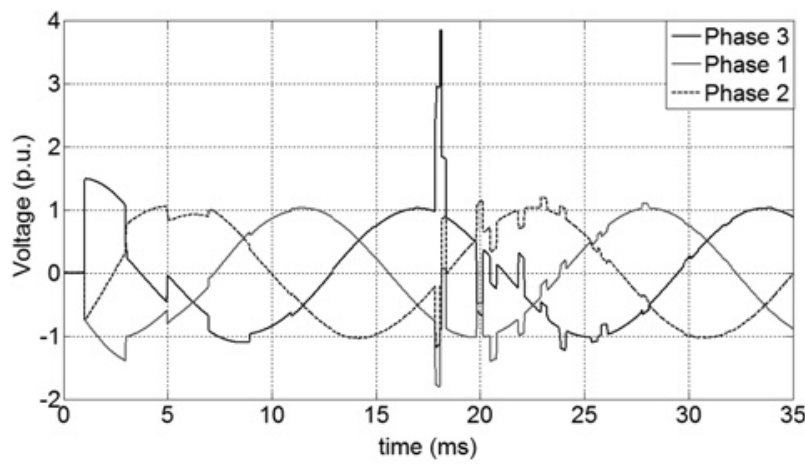

a
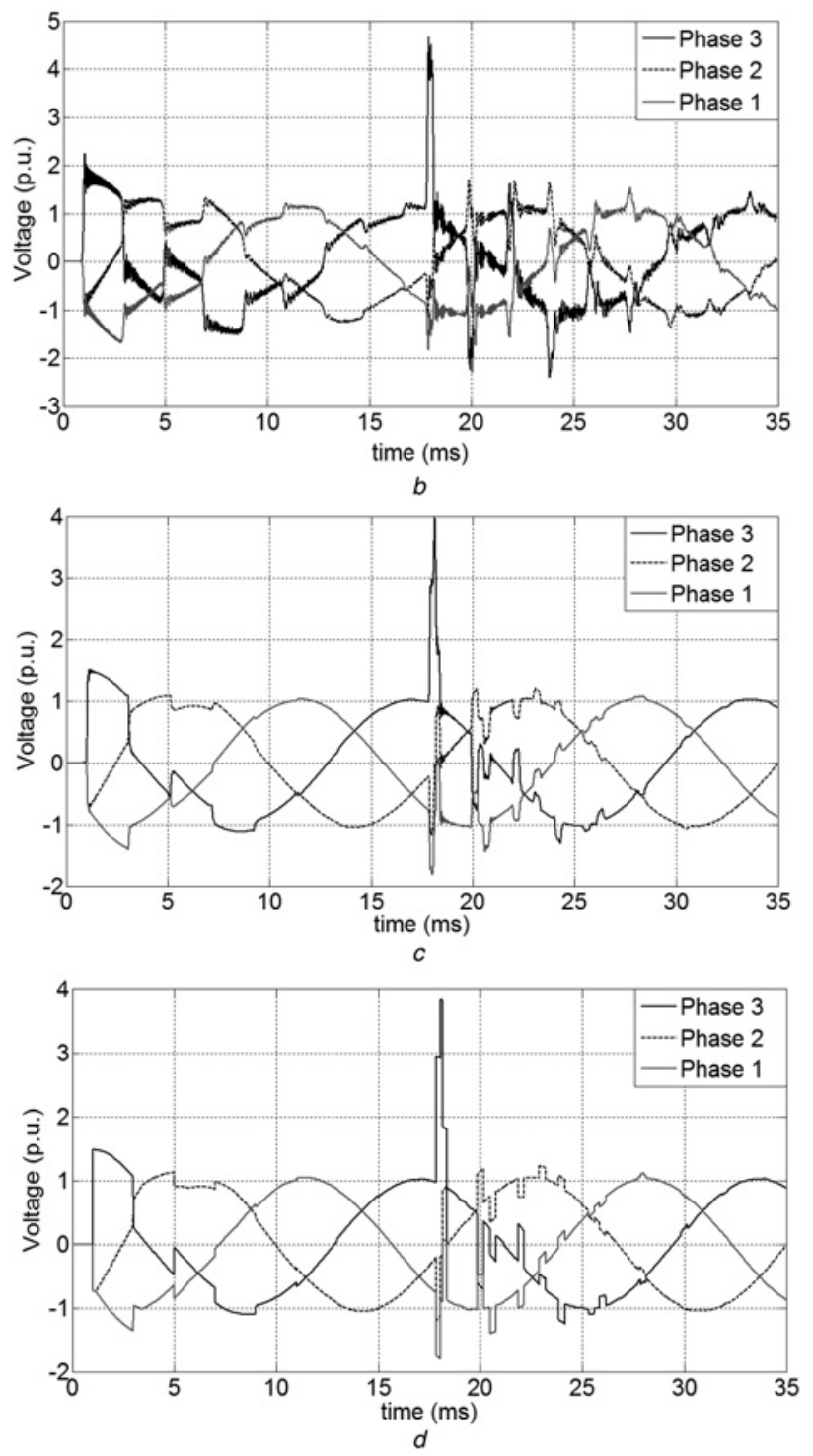

Fig. 10 Transient voltages at the receiving end of the three-phase transmission line

$a$ Modelling by NLT

$b$ Line model using lumped parameters without $\pi$ filter

$c$ Line model using lumped parameters with $\pi$ filter

$d$ Modelling by Bergeron

and voltage transients are calculated from three independent computational processes for each propagation mode, which are modelled as three single-phase lines by lumped parameters directly in the time domain. In sequence, the current and voltage transients are converted from the modes to phase values using a real and constant modal transformation matrix, such as Clarke's matrix $[7,8]$.
The proposed line model and filtering modelling are evaluated for a multiconductor system concerning a conventional $440 \mathrm{kV}$ transmission line with a single circuit, as described in Fig. 9a. This transmission line is designed with bundles with four conductors of type Groesbeck (phases 1,2 and 3) and two ground wires EHSW-3/8' (conductors 4 and 5). The phases are untransposed, and the line geometry is characterised by a symmetrical vertical plane. The soil resistivity is assumed to be $1000 \Omega \mathrm{m}$, and the line length is $150 \mathrm{~km}$. Fig. $9 b$ shows the untransposed transmission line with a synchronous generator connected at the sending end, whereas the receiving end is connected to a generic three-phase resistive load.

At time $t=0$, the sending end of the line is connected to the synchronous generator with 1 p.u. $/ 60 \mathrm{~Hz}$. After switching at the sending end, at $t=16.85 \mathrm{~ms}$ of the simulation, an impulsive voltage surge occurs at phase 3 , as represented by the voltage source $V(t)$ in Fig. $9 b$ and expressed in the following equation

$$
V(t)=\left\{\begin{array}{l}
0, \quad t \leq 16.85 \mathrm{~ms} \\
2 \text { p.u. }, \quad 16.85 \leq t \leq 17.15 \mathrm{~ms} \\
0, \quad t>17.15 \mathrm{~ms}
\end{array}\right.
$$

The electromagnetic transients are simulated using the line model using lumped elements with and without the inclusion of the proposed analogue $\pi$ filter to reduce the numerical oscillations (Figs. $10 b$ and $c$, respectively. The results are compared with the simulations obtained using the line model with NLT (Fig. 10a) and from the Bergeron method (Fig. 10d), available in the alternative transient program (ATP)/EMTP [3].

The voltage surge applied at phase 3 results in an electromagnetic transient at all three phases of the line. However, the numerical oscillations are significantly smoothed in the simulations obtained from the proposed line model with analogue $\pi$ filters, as observed in Fig. 10c, compared with the results of the simulations using the same line model without $\pi$ filters, as shown in Fig. 10b. The simulation obtained from the proposed line model with $\pi$ filters is similar to the results simulated using the line model with NLT and the Bergeron method. The results obtained from the four line models were calculated using the same numerical integration method and without variations in the integration step.

\section{Conclusions}

The inclusion of low-pass filters in the representation of a line by lumped parameters is shown to be a versatile and efficacious technique to suppress the numerical oscillations in electromagnetic transient simulations. Comparing the results obtained from the conventional line model by lumped parameters with and without $\pi$ filters shows a significant reduction in the erroneous oscillations, even compared with results obtained using NLT and the Bergeron method. In fact, the simulations conducted using the proposed modelling methodology and using the line model based on NLT are extremely similar, demonstrating that the proposed filtering technique provides very accurate results.

The same technique using analogue filters can be extended to other lumped-parameters models of power components and devices, e.g. power transformers and surge arresters, which are also approached in the technical literature by equivalent electric circuits in the time domain. In addition, the proposed method for suppressing numerical oscillations during electromagnetic transient simulations can be applied to more sophisticated models, using fitting techniques for representation of the frequency-dependent impedance directly in the time domain. These time-domain models are widely used for representation of various electromagnetic phenomena in power and telecom systems, from communication towers modelling to the lightning performance evaluation of power systems because of the easy inclusion of time-variable parameters and elements during transient simulations, which are usually a complex procedure in the frequency domain. 


\section{Acknowledgments}

This research was financially supported by grants from the São Paulo Research Foundation - FAPESP (Proc. 12/13857 and 15/10204-8) and National Council for Scientific and Technological Development - CNPq.

\section{References}

1 Umoto, J., Hara, T.: 'Numeric analysis of surge propagation on single-conductor systems considering corona losses', Electr. Eng. Jpn, 1969, 1, pp. 21-28

2 Mamis, M.S.: 'Computation of electromagnetic transients on transmission lines with nonlinear components', IEE Proc. Gener. Transm. Distrib., 2003, 150, (2), pp. 200-204

3 Dommel, H.W.: 'EMTP theory book' (Vancouver, 1986)

4 Jena, M.K., Samantaray, S.R.: 'Intelligent relaying scheme for series-compensated double circuit lines using phase angle of differential impedance', Int. J. Electr. Power Energy Syst., 2015, 70, pp. 17-26

5 Macías, J.A.R., Expósito, A.G., Soler, A.B.: 'A comparison of techniques for state-space transient analysis of transmission lines', IEEE Trans. Power Deliv., 2005, 20, (2), pp. 894-903

6 Mamis, M.S., Koksal, M.: 'Solution of eigenproblems for state-space transient analysis of transmission lines', Electr. Power Syst. Res., 2000, 55, pp. 7-14

7 Costa, E.C.M., Kurokawa, S., Pinto, A.J.G., et al.: 'Simplified computational routine to correct the modal decoupling in transmission lines and power systems modelling', IET Sci. Meas. Technol., 2013, 7, (1), pp. 7-15

8 Costa, E.C.M., Kurokawa, S., Pissolato, J., et al.: 'Efficient procedure to evaluate electromagnetic transients on three-phase transmission lines', IET Gener. Transm. Distrib., 2010, 4, pp. 1069-1081
9 Mamis, M.S., Koksal, M.: 'Remark on the lumped parameter modeling of transmission lines', Electr. Mach. Power Syst., 2000, 28, (6), pp. 565-575

10 Siqueira, J.C.G., Bonatto, B.D., Marti, J., et al.: 'A discussion about optimum time step size and maximum simulation time in EMTP-based programs', Int. J. Electr. Power Energy Syst., 2015, 72, pp. 24-32

11 Chrysochos, A.I., Tsolaridis, G., Papadopoulos, T., et al.: 'Damping of oscillations related to lumped-parameter transmission line modeling'. Proc. of Int. Conf. on Power Systems Transients - IPST'15, Croatia, June 2015

12 Costa, E.C.M., Kurokawa, S., Shinoda, A.A., et al.: 'Digital filtering of oscillations intrinsic to transmission line modeling based on lumped parameters', Int. J. Electr. Power Energy Syst., 2013, 44, pp. 908-915

13 Merwe, J., Cloete, J.H., Reader, H.C.: 'Transients on multiconductor transmission lines above dissipative earth-numerical simulation and measurement', IEEE Trans. Electromagn. Compat., 2003, 45, (2), pp. 404-415

14 Zhang, Y., Spielman, B.E.: 'A stability analysis for time-domain method-of-moments analysis of 1-D double-negative transmission lines', IEEE Trans. Microw. Theory Tech., 2007, 55, (9), pp. 1887-1898

15 Ramirez, A., Semlyen, A., Iravani, R.: 'Modeling nonuniform transmission lines for time domain simulation of electromagnetic transients', IEEE Trans. Power Deliv., 2003, 18, (3), pp. 968-974

16 Jurić-Grgić, V., Lucić, R., Bernadić, A.: 'Transient analysis of coupled non-uniform transmission line using finite element method', Int. J. Circuit Theory Appl., 2014, 43, (9), pp. 1167-1174

17 Lucić, R., Jurić-Grgić, I., Kurtović, M.: 'Time domain finite element method analysis of multi conductor transmission lines', Int. Trans. Electr. Energy Syst., 2010, 20, (6), pp. 822-832

18 Moreno, P., Ramirez, A. 'Implementation of the numerical Laplace transform: a review', IEEE Trans. Power Deliv., 2008, 8, (4), pp. 2599-2609

19 Gómez, P., Uribe, F.A.: 'The numerical Laplace transform: an accurate technique for analyzing of electromagnetic transients on power system devices', Int. J. Electr. Power Energy Syst., 2009, 31, (2-3), pp. 116-123

20 Huelsman, L.P.: 'Active and passive analog filter design: an introduction' (Mcgraw-Hill, 1993) 